\title{
Hypertension and prolonged vasoconstrictor signaling in RGS2-deficient mice
}

\author{
Scott P. Heximer, ${ }^{1}$ Russell H. Knutsen, ${ }^{1}$ Xiaoguang Sun, ${ }^{1}$ Kevin M. Kaltenbronn, ${ }^{1}$ \\ Man-Hee Rhee, ${ }^{1}$ Ning Peng, ${ }^{2}$ Antonio Oliveira-dos-Santos, ${ }^{3}$ Josef M. Penninger, ${ }^{3}$ \\ Anthony J. Muslin, ${ }^{1,4}$ Thomas H. Steinberg, ${ }^{1,5}$ J. Michael Wyss, ${ }^{2}$ Robert P. Mecham, ${ }^{1}$ \\ and Kendall J. Blumer ${ }^{1}$ \\ ${ }^{1}$ Department of Cell Biology and Physiology, Washington University School of Medicine, St. Louis, Missouri, USA \\ ${ }^{2}$ Department of Cell Biology, University of Alabama at Birmingham, Birmingham, Alabama, USA \\ ${ }^{3}$ Amgen Institute, Toronto, Ontario, Canada \\ ${ }^{4}$ Division of Cardiology, and \\ ${ }^{5}$ Department of Internal Medicine, Washington University School of Medicine, St. Louis, Missouri, USA
}

\begin{abstract}
Signaling by hormones and neurotransmitters that activate $G$ protein-coupled receptors (GPCRs) maintains blood pressure within the normal range despite large changes in cardiac output that can occur within seconds. This implies that blood pressure regulation requires precise kinetic control of GPCR signaling. To test this hypothesis, we analyzed mice deficient in RGS2, a GTPase-activating protein that greatly accelerates the deactivation rate of heterotrimeric $\mathrm{G}$ proteins in vitro. Both $\mathrm{rgs} \mathrm{2}^{+/-}$ and $r g s 2^{-/-}$mice exhibited a strong hypertensive phenotype, renovascular abnormalities, persistent constriction of the resistance vasculature, and prolonged response of the vasculature to vasoconstrictors in vivo. Analysis of $\mathrm{P} 2 \mathrm{Y}$ receptor-mediated $\mathrm{Ca}^{2+}$ signaling in vascular smooth muscle cells in vitro indicated that loss of RGS2 increased agonist potency and efficacy and slowed the kinetics of signal termination. These results establish that abnormally prolonged signaling by $\mathrm{G}$ protein-coupled vasoconstrictor receptors can contribute to the onset of hypertension, and they suggest that genetic defects affecting the function or expression of RGS2 may be novel risk factors for development of hypertension in humans.
\end{abstract}

J. Clin. Invest. 111:445-452 (2003). doi:10.1172/JCI200315598.

\section{Introduction}

Signaling by $G$ protein-coupled neurotransmitter receptors in the autonomic nervous system and vasoregulatory factor receptors in the periphery govern blood pressure by controlling constriction and dilatation of resistance arterioles, and electrolyte and fluid balance by the kidney $(1,2)$. Hypertension can be caused in rodents by deletion of genes encoding $G$ protein-coupled receptors (GPCRs) that normally decrease blood pressure $(3,4)$, or by overproduction of GPCR agonists that elevate blood pressure $(5,6)$. Increased risk of hypertension in certain human populations has been

Received for publication April 3, 2002, and accepted in revised form November 14, 2002.

Address correspondence to: Kendall J. Blumer, Department of Cell Biology and Physiology, Washington University

School of Medicine, 660 South Euclid Avenue, St. Louis,

Missouri 63110, USA. Phone: (314) 362-1668;

Fax: (314) 362-7463; E-mail: kblumer@cellbio.wustl.edu.

Scott P. Heximer's present address is: Department of Physiology,

University of Toronto, Toronto, Ontario, Canada.

Conflict of interest: The authors have declared that no conflict of interest exists.

Nonstandard abbreviations used: G protein-coupled receptor (GPCR); regulator of $G$ protein signaling (RGS); polyethylene (PE); angiotensin II type 1 (AT1); PBS-0.04\% Tween-20 (PBS-T); mean arterial pressure (MAP). associated with genetic polymorphisms in genes encoding components of GPCR signaling pathways (7-13).

Several considerations have led us to hypothesize that normal blood pressure homeostasis requires the function of newly identified kinetic regulatory components of GPCR signaling systems, the RGS proteins (regulators of $\underline{G}$ protein signaling). When cardiac output changes rapidly in response to metabolic demand or arousal state, systemic blood pressure remains within the normal range. This implies that the GPCR signaling pathways controlling blood pressure must be activated and deactivated rapidly to compensate for large changes in cardiac output that can occur within seconds. Consistent with this concept, G protein activation by receptor-catalyzed guanine nucleotide exchange is fast (less than 1 second) (14). In contrast, $G$ protein deactivation rates determined by the intrinsic ability of $\mathrm{G} \alpha$ subunits to hydrolyze GTP are often much slower (half-time $\sim 30$ seconds) than deactivation rates in vivo inferred from physiological measurements (15). However, $G$ protein deactivation in vitro can be stimulated greatly by members of the RGS family of proteins, which are potent GTPase-activating proteins for $G \alpha$ subunits (15). We therefore hypothesized that mouse mutants lacking relevant RGS proteins would have abnormal blood pressure 
due to prolonged signaling by GPCR signaling pathways involved in blood pressure control.

Of the approximately 30 RGS proteins encoded in the mouse or human genome, RGS2 may be critical for blood pressure regulation. Among RGS proteins examined thus far in detail, RGS2 appears to be the most potent negative regulator of $\mathrm{Gq} \alpha$, which mediates the action of most physiological vasoconstrictors, including norepinephrine, angiotensin II, endothelin-1, and thrombin. RGS2 also can attenuate Gi- and Gs-mediated pathways $(16,17)$, which could affect blood pressure regulation by other physiologically important agonists such as serotonin, dopamine, or bradykinin. The rgs 2 gene is expressed in tissues and cell types involved in blood pressure regulation, including the nervous system $(16,18,19)$, kidney $(18)$, and vascular smooth muscle cells, where its expression is induced by angiotensin II as a potential inhibitory feedback mechanism (20). Lastly, the human rgs2 gene maps to 1q31 (21), within an interval (1q31-42) linked to one of three allelic forms of an inherited human hypertension disorder (22).

To determine whether RGS2 is required for normal blood pressure homeostasis, we have analyzed mice lacking a functional rgs2 gene. Previous studies have shown that RGS2 deficiency in mice has no apparent effect on motor responses, circadian activity, exploratory behavior, motor coordination, or spatial learning and memory; however, loss of RGS2 does cause partial defects in T cell activation, a male-specific aggression deficit, and decreased density of apical and basilar spines in the CA1 region of the hippocampus (19). Here we report that both $r g s 2^{+/-}$and $r g s 2^{-/-}$mice are strongly hypertensive. Our pharmacological and physiological analysis of these mutants and studies of signal transduction in vascular smooth muscle cells derived from them indicate that precise kinetic regulation of vasoconstrictor signaling is required for normal blood pressure homeostasis.

\section{Methods}

Mice. The generation and genotyping of $r g s 2^{-/-}$mice have been described (19). All animals were bred $(n>10)$ into a C57BL/ 6 background. Mice used in this study were provided ad libitum with normal food and water and subjected to a standard light/dark cycle. All experiments were performed in accordance with Animal Studies Committee regulations at Washington University School of Medicine.

Blood pressure analysis and echocardiography. Mean arterial pressures (MAPs) of mice anesthetized with ketamine (87 $\mathrm{mg} / \mathrm{kg}$ ) and xylazine $(13 \mathrm{mg} / \mathrm{kg})(23)$ were recorded by catheterization of the common carotid artery with a Millar 1.4 French blood pressure probe (Millar Instruments Inc., Houston, Texas, USA) that was passed into the ascending aorta. All drugs were diluted in $0.9 \%$ sodium chloride. Drugs were administered ( $5 \mu \mathrm{l}$ doses added $1-2$ $\mu \mathrm{l} / \mathrm{s})$ at the indicated concentrations by catheterization of the left jugular vein with polyethylene tubing (PE-10) attached to a $1-\mathrm{ml}$ syringe. In experiments where $\alpha$-adrenergic or angio-tensin II type 1 receptor antagonists were used, we routinely established that the blockade was effective by showing that a bolus injection of the relevant specific agonist had no effect. Ganglionic blockade by hexamethonium was verified as follows. Under ketamine/xylazine anesthesia there are occasional episodes of increased parasympathetic tone marked by bradycardia accompanied by abnormally low blood pressures. Such episodes were extinguished within seconds of administration of hexamethonium, which normalized heart rate and blood pressure, indicating complete autonomic blockade. For analysis of blood pressure in waking animals (24), mice subjected to isoflurane anesthesia were catheterized through the femoral artery with $0.05 \mathrm{~mm}$ tapered PE tubing. Catheters were tunneled through the skin at the nape of the neck and fixed in place with dental acrylic. Direct arterial pressures were digitally monitored the next morning in awake, freely moving mice. Normal baroreflex responses, indicated by an appropriate heart rate decrease, were recorded following a phenylephrine challenge $(6 \mu \mathrm{g} / \mathrm{kg}$ intra-arterially). PE-tubing catheters were similarly implanted in the right carotid artery of selected mice to confirm similarly high blood pressures in the ascending aorta. M-mode echocardiography was performed as de-scribed previously (25). The reported data for each experimental condition were mean values obtained from three to ten mice, which were analyzed for statistical significance by Student's $t$ test.

Histology and morphometric analysis of renal vasculature. Mice were perfused at constant pressure $(140 \mathrm{mmHg})$ with $10 \%$ phosphate-buffered formalin. Sections of paraffin-embedded tissues were prepared, and stained with Verhoeff-Van Gieson reagent (elastin stain) or hematoxylin and eosin. We performed morphometric analysis of medial thickness in renal resistance vessels with diameters of $50-100 \mu \mathrm{m}$ by analyzing 30 vessels obtained from at least two mice of each genotype.

Immunoblotting analysis of angiotensin II type 1 receptor levels in aortic lysates. Angiotensin II type 1 (AT1) receptor levels in aortic protein extracts from wild-type rats and $r g s 2^{+/+}, r g s 2^{+/-}$, and $r g s 2^{-/-}$mice were analyzed by immunoblotting experiments. Thoracic aortae were homogenized in RIPA buffer $(150 \mathrm{mM} \mathrm{NaCl}, 1.0 \%$ IGEPAL, 0.5\% deoxycholate [Sigma-Aldrich, St. Louis, Missouri, USA], $0.1 \%$ SDS, $50 \mathrm{mM}$ Tris-HCl, $\mathrm{pH}$ 8.0) containing protease inhibitors. Thirty micrograms of total protein per lane was resolved by PAGE and transferred to PVDF membranes. Membranes were blocked overnight at $4^{\circ} \mathrm{C}$ in PBS- $0.04 \%$ Tween-20 (PBS-T) containing $5 \%$ nonfat dry milk. For detection of AT1 receptor, membranes were incubated with affinitypurified anti-AT1 receptor antibody (Santa Cruz Biotechnology Inc., Santa Cruz, California, USA) diluted 1:1,000 in PBS-T with $0.5 \%$ nonfat dry milk and goat anti-rabbit horseradish peroxidase-conjugated secondary antibody (ICN Biomedicals Inc., Costa Mesa, California, USA) diluted 1:2,000 in PBS-T. AT1 receptor protein was detected by enhanced chemiluminescence. For peptide blocking studies, the diluted anti-AT1 receptor antibody was incubated overnight 
with a 700-fold molar excess of blocking peptide (Santa Cruz Biotechnology Inc.) in PBS, and samples were processed as described above.

Measurement of agonist-induced changes in intracellular calcium in primary aortic vascular smooth muscle cell cultures. Vascular smooth muscle cells from explanted thoracic aortic tissue were prepared as described (26), with the following modifications. Cells were dissociated by gentle agitation for 30 minutes at $37^{\circ} \mathrm{C}$ in DMEM digestion buffer containing elastase $(0.03 \%$ $\mathrm{wt} / \mathrm{vol})$ and collagenase $(0.07 \% \mathrm{wt} / \mathrm{vol})$, sieved through 50- $\mu \mathrm{m}$ nylon mesh, washed, and seeded at low density onto polylysine-coated $13-\mathrm{mm}$ no. 1 glass coverslips. Cells in culture were maintained in DMEM/F12 media containing 20\% FCS and $2 \mathrm{mM}$ glutamine with penicillin and streptomycin. Immunostaining with anti-smooth muscle $\alpha$-actin antibody (1A4; Sigma-Aldrich) indicated that cell preparations were more than $80 \%$ vascular smooth muscle cells. Cells were washed and incubated in $4 \mu \mathrm{M}$ fura-2/AM (Molecular Probes Inc., Eugene, Oregon, USA) in Ca1 solution ( $11 \mathrm{mM}$ glucose, $130 \mathrm{mM} \mathrm{NaCl}$, $4.8 \mathrm{mM} \mathrm{KCl}, 1.2 \mathrm{mM} \mathrm{MgCl}$, $17 \mathrm{mM}$ HEPES, $1 \mathrm{mM}$ $\mathrm{CaCl}_{2}, \mathrm{pH} 7.3$ ) for 30 minutes at $37^{\circ} \mathrm{C}$, washed once, and incubated in $\mathrm{Ca} 1$ without fura- 2 for 10 minutes at $37^{\circ} \mathrm{C}$ to allow hydrolysis of the acetoxymethyl ester. Coverslips were placed in an RC-26G large open bath recording chamber (Warner Instrument Corp., Hamden, Connecticut, USA) that was mounted on a Nikon Eclipse E600 FN microscope equipped with a Nikon Fluor $40 \times / 0.80$ water immersion lens (Nikon Inc., Melville, New York, USA). Excitation light (340 $\mathrm{nm} / 380 \mathrm{~nm}$ ) was provided by a TiLL Polychrome IV monochromater (TiLL Photonics GmbH, Martinsried, Germany) in conjunction with a 495-nm dichroic mirror and a $525 \pm 20-\mathrm{nm}$ emission filter (Chroma Technology Corp., Brattleboro, Vermont, USA). Fluorescence ratio imaging was performed with TiLLvisION 4.0 imaging software (TiLL Photonics $\mathrm{GmbH}$ ), and images were acquired with a SensiCam cooled CCD camera (PCO Computer Optics GmbH, Kelheim, Germany). Image pairs were taken every 2 seconds, and background-corrected ratio images (340 $\mathrm{nm} / 380 \mathrm{~nm}$ ) were analyzed with individual cells defined as regions of interest (ROIs). Data are presented as relative ratio values over time for each ROI.

\section{Results}

We determined whether RGS2 is required for normal blood pressure homeostasis by measuring aortic blood pressure (23) of anesthetized wild-type, $\mathrm{rgs}_{\mathrm{s} 2^{+/-}}$, and $\mathrm{rgs}_{\mathrm{s} \mathrm{2}^{-/-}}$ mice. The MAP of the rgs $2^{-/-}$mutant was $135 \pm 3 \mathrm{mmHg}$, compared with $84 \pm 6 \mathrm{mmHg}$ for isogenic wild-type controls, indicating a large elevation in blood pressure $(\sim 50$ $\mathrm{mmHg}$; Figure 1). Strikingly, mice heterozygous for an rgs2 deletion exhibited a similar level of hypertension ( $\mathrm{MAP}=134 \pm 3 \mathrm{mmHg}$; Figure 1), indicating that RGS2 is especially critical for blood pressure homeostasis. Such a result has a precedent, since blood pressures of mice heterozygous or homozygous for a deletion of the dopamine D3 receptor gene are elevated to the same extent even though renin levels are higher in the homozygote (3). The hypertensive phenotype of $\mathrm{rgs}^{2-{ }^{--}}$ mutants was confirmed in unanesthetized freely moving mice (24) (Table 1), which indicated a hypertensive phenotype significantly greater than that observed in established mouse models of hypertension, including the uninephrectomized deoxycorticosterone acetate-salt model (27) and GPCR knockout models $(3,4)$.

RGS2-deficient mice exhibited other cardiovascular phenotypes similar to some of those in humans with chronic essential hypertension (28). Significant renovascular abnormalities (medial thickening indicative of vascular smooth muscle cell hypertrophy and/or hyperproliferation) were also observed in RGS2-deficient mice (Figure 2). Transthoracic echocardiography, however, indicated that, relative to wild-type mice of the same age, 6-month-old RGS2-deficient mice exhibited no significant decrease in systolic contractile function as measured by percent fractional shortening (Table 2) or evidence of hypertrophy as indicated by various cardiac dimensions (Table 2) or heart/body weight ratios (data not shown).

To investigate the mechanisms responsible for the hypertensive phenotype of rgs 2 mutants, we initially determined whether these mutants exhibited increased pressor responses following vasoconstrictor challenge (29), as can occur in hypertensive humans due to hypertrophy of resistance arterioles (28). Compared with wild-type controls, RGS2-deficient mice displayed small increases in blood pressure in response to a maximal dose of angiotensin II (Figure 3a) or phenylephrine (data not shown), indicating that hypertrophy of the resistance vasculature was not the main cause of the hypertensive phenotype. Therefore, the hypertensive phenotype of $r g s 2$ mutants was likely to be caused by increased vascular tone (chronic constriction of the peripheral vasculature) in response to signaling via neurotransmitters or circulating factors, or by abnormal fluid and electrolyte balance.

Increased neurotransmitter signaling resulting in elevated vascular tone was unlikely to be the cause of the observed hypertensive phenotype, because the anesthesia

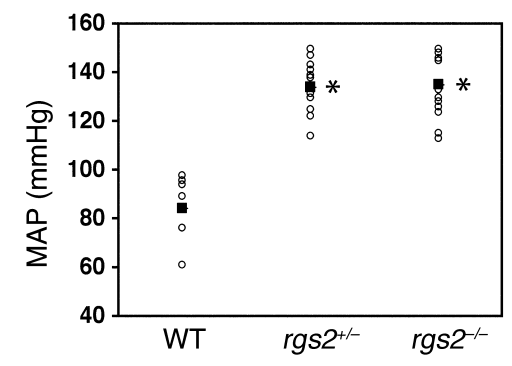

\section{Figure 1}

Blood pressures of wild-type and RGS2-deficient mice. MAPs $(\mathrm{mmHg}$; open circles) of anesthetized male wild-type (WT; $n=8), \operatorname{rgs} 2^{+/-}(n=13)$, and $r g s 2^{-/-}(n=15)$ mice with heart rates greater than 330 beats per minute are shown. The mean blood pressure for each genotype is indicated (filled squares). ${ }^{*} P<0.0005$ relative to wild-type mice. 


\section{Table 1}

Blood pressures and heart rates of unanesthetized mice, and of anesthetized mice following nicotinic, $\alpha$-adrenergic, or AT1 receptor blockade

\begin{tabular}{lcccc}
\hline & \multicolumn{2}{c}{ Wild-type } & \multicolumn{2}{c}{ rgs2-/- } \\
& MAP & HR & MAP & HR \\
Unanesthetized & $100.4 \pm 2.5^{\mathrm{A}}$ & $530 \pm 31$ & $125 \pm 2.5^{\mathrm{A}}$ & $514 \pm 8$ \\
Anesthetized & & & & \\
Hexamethonium & & & & \\
- & $76.5 \pm 2.6$ & $309 \pm 19$ & $128.5 \pm 4.1$ & $341 \pm 23$ \\
+ & $89.2 \pm 7.4$ & $341 \pm 9$ & $125.3 \pm 4.6$ & $372 \pm 24$ \\
Hexamethonium + & prazosin & & & \\
- & $97.3 \pm 1.3$ & $348 \pm 22$ & $128.7 \pm 3.1^{\mathrm{B}}$ & $398 \pm 31$ \\
+ & $82.9 \pm 5.8$ & $335 \pm 15$ & $115.6 \pm 2.3^{\mathrm{B}}$ & $395 \pm 31$ \\
Candesartan & & & & \\
- & $86.1 \pm 2.8^{\mathrm{C}}$ & $290 \pm 28$ & $137.8 \pm 8.0^{\mathrm{C}}$ & $367 \pm 4$ \\
+ & $68.1 \pm 3.4^{\mathrm{C}}$ & $287 \pm 28$ & $81.2 \pm 5.2^{\mathrm{C}}$ & $293 \pm 20$ \\
Hexamethonium + candesartan & & & \\
- & $81.1 \pm 3.2^{\mathrm{A}}$ & $319 \pm 16$ & $131.5 \pm 4.8^{\mathrm{D}}$ & $348 \pm 17$ \\
+ & $68.7 \pm 2.9^{\mathrm{A}}$ & $345 \pm 7$ & $85.4 \pm 3.5^{\mathrm{D}}$ & $334 \pm 24$
\end{tabular}

Mean \pm SEM of results from experiments with three to five mice under each condition. Paired blood pressure values (i.e., unanesthetized wild-type vs. rgs $2^{-/}$mice; anesthetized mice of a given genotype with or without the indicated drugs) that were statistically different are indicated ( ${ }^{A} P<0.02$, $\left.{ }^{B} P<0.05, C P<0.01, D P<0.00005\right)$. Differences in heart rates were not statistically significant. HR, heart rate.

employed in the preceding experiments inhibits sympathetic outflow caused by activation of inhibitory $\alpha_{2 A^{-}}$ adrenergic receptors. Furthermore, similar to rats subjected to the same anesthesia protocol (30), blocking ganglionic transmission with hexamethonium, an inhibitor of nicotinic channels, had little effect on MAP of wild-type or RGS2-deficient mice (Table 1). However, because not all sympathoexcitatory signals are blocked by hexamethonium (31), we determined whether the hypertensive phenotype depends on activation of peripheral $\alpha_{1}$-adrenergic receptors, the primary mediators of adrenergic vasopressor responses (32). After administration of hexamethonium, blockade of $\alpha_{1}$-adrenergic receptors with prazosin reduced MAP modestly and to a similar extent $(\sim 13-15 \mathrm{mmHg})$ in wild-type and RGS2-deficient mice (Table 1). Therefore, signaling via prazosin-sensitive $\alpha$-adrenergic receptors did not seem to be required to detect a hypertensive phenotype in RGS2-deficient mice.

In contrast, the hypertensive phenotype of RGS2deficient mice was profoundly dependent on angiotensin II signaling. Angiotensin II, the effector of the renin-angiotensin system, controls blood pressure

\section{Figure 2}

Structures of aorta and renal vasculature in wild-type and RGS2-deficient mice. Histological analysis (hematoxylin-and-eosin or Verhoeff-Van Gieson elastin staining) of wild-type (a, c, and $\mathbf{e})$ and $r g s 2^{-l_{-}}$(b, $\mathbf{d}$, and $\mathbf{f}$ ) mice. Aorta ( $\mathbf{a}$ and $\mathbf{b}$ ), renal interlobular arteries ( $\mathbf{c}$ and $\mathbf{d}$ ), and nonelastic arterioles of the renal cortex (e and $\mathbf{f}$ ) are shown. (g) Morphometric analysis of relative medial thickness (medial area/total area) of renal resistance vessels with diameters of $50-100 \mu \mathrm{m}$ from wild-type, rgs $2^{+/-}$, and $r g s 2^{-/-}$mice. Relative medial thickness of $r g s 2$ mutants differed significantly $\left({ }^{*} P<0.0001\right)$ from that of wild-type mice. by activating $\mathrm{G}$ protein-coupled AT1 receptors that stimulate constriction of the resistance vasculature to regulate vascular tone, and that stimulate aldosterone production by the adrenal medulla to regulate electrolyte and water balance $(1,2)$. Infusion of an AT1 receptor antagonist (candesartan) dramatically decreased blood pressure in RGS2-deficient mice (Table 1 and Figure $3 \mathrm{~b}$ ). AT1 receptor blockade in rgs $2^{-/-}$mice decreased blood pressure to base-line values within a few minutes (data not shown), suggesting that hypertension was probably not caused by significant effects on fluid or electrolyte balance. Therefore, hypertension in $\operatorname{rgs}^{-/-}$mice was likely caused by angiotensin II-dependent increases in vascular tone.

We obtained further evidence of increased vascular tone in RGS2-deficient mice by measuring rates of blood pressure decline after infusion of an AT1 receptor antagonist. AT1 receptor blockade resulted in a slower decrease in blood pressure in $r g s 2^{--}$mice than in wild-type controls (Figure 3b). A similar result was obtained under conditions where initial blood pressures were established at similar high levels in RGS2-deficient and wild-type mice by pretreatment with a pressor dose of angiotensin II. Under these conditions, subsequent infusion of an AT1 receptor antagonist caused blood pressure to decrease significantly less rapidly in $r g s 2^{-/-}$mutants than in wildtype controls (Figure 3c). One potential explanation for
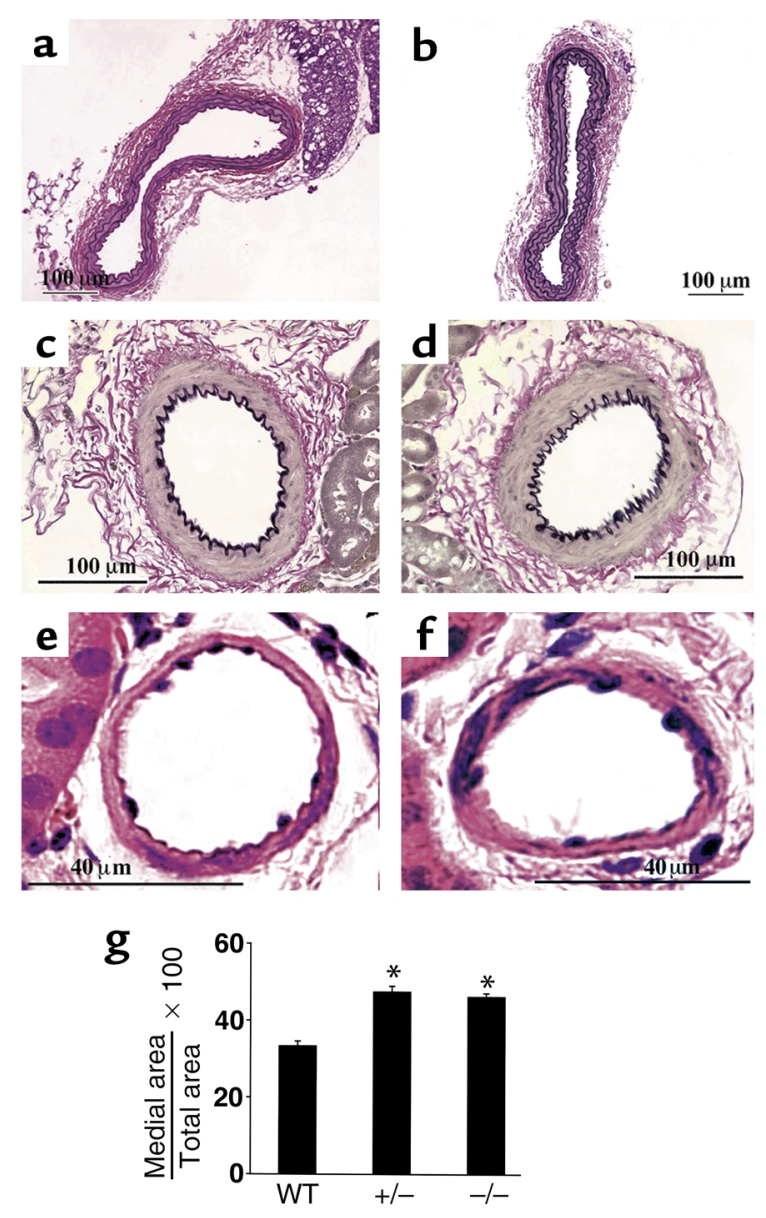
Table 2

Echocardiographic analysis of wild-type and RGS2-deficient mice

\begin{tabular}{|c|c|c|c|}
\hline & Wild-type & $\operatorname{rgs} 2^{+/-}$ & $r g s 2^{-/-}$ \\
\hline Heart rate (bpm) & $643 \pm 27$ & $647 \pm 10$ & $639 \pm 11$ \\
\hline LVPWd (mm) & $0.82 \pm 0.05$ & $0.85 \pm 0.02$ & $0.87 \pm 0.03$ \\
\hline IVSd (mm) & $0.85 \pm 0.05$ & $0.88 \pm 0.03$ & $0.90 \pm 0.04$ \\
\hline LVIDd (mm) & $3.43 \pm 0.12$ & $3.39 \pm 0.14$ & $3.38 \pm 0.07$ \\
\hline LVPWs (mm) & $1.58 \pm 0.04$ & $1.55 \pm 0.05$ & $1.63 \pm 0.07$ \\
\hline IVSs (mm) & $1.62 \pm 0.04$ & $1.57 \pm 0.03$ & $1.62 \pm 0.07$ \\
\hline LVIDs (mm) & $1.69 \pm 0.11$ & $1.70 \pm 0.13$ & $1.62 \pm 0.07$ \\
\hline FS (\%) & $51.0 \pm 1.8$ & $50.2 \pm 1.9$ & $52.2 \pm 1.4$ \\
\hline
\end{tabular}

Cardiac dimensions: LV, left ventricle; LVPWd, LV posterior wall, diastole; IVSd, interventricular septum, diastole; LVIDd, LV internal dimension, diastole; LVPWs, LV posterior wall, systole; IVSs, interventricular septum, systole; LVIDs, LV internal dimension, systole; FS, fractional shortening. Dimensions were obtained from six to seven mice for each genotype. FS was calculated as $100 \% \times[($ LVIDd - LVIDs $) /$ LVIDd $]$. Data shown are the mean \pm SEM. bpm, beats per minute.

the angiotensin II-dependent hypertensive phenotype was elevated vascular expression of AT1 receptors in RGS2-deficient mice. However, AT1 receptor levels in aortae from wild-type, $r g s 2^{+/-}$, and $r g s 2^{-/-}$mice were indistinguishable (Figure $3 \mathrm{~d}$ ), arguing against this hypothesis. Therefore, the results suggested that RGS2 regulates vascular tone by contributing to attenuation of AT 1 receptor signaling in the resistance vasculature.

Results of additional experiments indicated that loss of RGS2 results in prolonged responses to other vasoconstrictor agonists. For these experiments, hexamethonium was used to block ganglionic transmission, and an AT1 receptor antagonist was administered to reduce blood pressure of wild-type and RGS2-deficient mice to similar low levels. Mice then were challenged with increasing doses of phenylephrine, a potent vasoconstrictor that activates Gq-coupled $\alpha_{1}$-adrenergic receptors in vascular smooth muscle cells. At each dose of phenylephrine, the increase in blood pressure was only slightly higher in $r g s 2^{-/-}$than in wild-type mice (Figure 4a), indicating that vascular sensitivity to this agonist was only slightly increased. In contrast, after phenylephrine challenge, the blood pressure of $r g s 2^{-/-}$ mice declined significantly more slowly than that of wild-type mice (i.e., termination of vasoconstrictor responses was slower; Figure 4b). These results suggested that a defect in vasoconstrictor-signal termination in cells of the peripheral vasculature contributed to the hypertensive phenotype of $r g s 2^{-/-}$mice.

To determine directly whether loss of RGS2 causes a defect in the termination of vasoconstrictor signaling, we examined vasoconstrictor signaling in aortic smooth muscle cells cultured from wild-type and rgs $2^{-{ }^{-}}$ mice. Responses of cells to stimulation of Gq-coupled P2Y receptors with ATP (a potent vasoconstrictor) were assessed by fura-2 imaging of intracellular calcium levels (Figure 5). As indicated by experiments that measured peak responses elicited by various concentrations of ATP (cultured vascular smooth muscle cells do not express P2X receptors; ref. 33), cells from $r g s 2^{-/-}$mice were approximately threefold more sensitive than cells from wild-type mice (Figure 5a). Furthermore, at the highest doses of ATP, peak calcium responses were greater in cells from $r g s 2^{-/-}$compared with wild-type mice, indicating that both the potency and the efficacy of ATP were greater. However, potency and efficacy of $\alpha$-adrenergic agonists on vasoconstriction in vivo were unaffected by loss of RGS2; this could be due to buffering by endothelial tone or other factors in vivo.

Fura-2 imaging of intracellular calcium levels was also used to examine the kinetics of signal termination following the peak response elicited by challenge with a maximal dose of ATP. As indicated by the kinetics with which intracellular $\mathrm{Ca}^{2+}$ levels declined to baseline levels after reaching a peak response, the signal termination kinetics of aortic smooth muscle cells from RGS2-deficient mice was markedly slower (Figure $5 \mathrm{~b}$ ). The results of these in vitro studies and analogous studies of the termination of vasoconstrictor

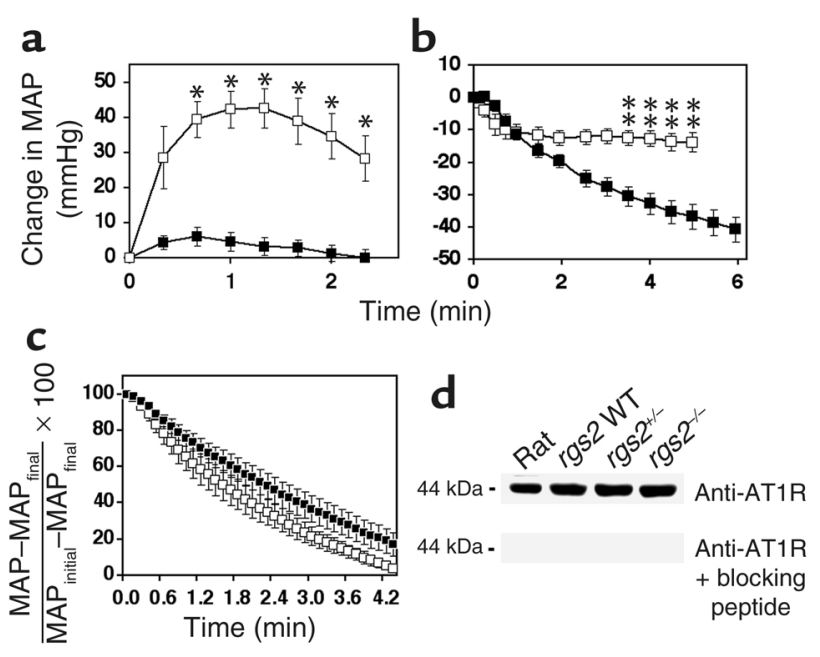

\section{Figure 3}

Blood pressure responses following challenge with angiotensin II or AT1 receptor blockade in wild-type and RGS2-deficient mice. (a) Time courses of blood pressure increase (change in MAP \pm SEM, $\mathrm{mmHg}$ ) following intraarterial administration of pressor doses of angiotensin II to RGS2-deficient mice ( $n=6$; filled squares) and wildtype mice ( $n=6$; open squares). (b) Time courses of blood pressure decrease (change in MAP $\pm \mathrm{SEM}, \mathrm{mmHg}$ ) following AT1 receptor blockade with candesartan in wild-type ( $n=6$; open squares) and rgs $2^{-1-}$ mice ( $n=6$; filled squares). (c) Time course of blood pressure decrease $\left[\left(M A P-M A P_{\text {final }}\right) /\left(M A P_{\text {initial }}-M P_{\text {final }}\right)\right] \pm S E M$ following AT1 receptor blockade in wild-type ( $n=7$; open squares) and rgs $2^{-1-}$ mice ( $n=7$; filled squares) that were pretreated with a pressor dose of angiotensin II to elevate blood pressures to similarly high starting values (systolic blood pressure $=160-170 \mathrm{mmHg}$ ). The results shown in each panel are representative of more than ten experiments performed with each genotype. The changes in blood pressure for wildtype and rgs $2^{-1-}$ mice were compared at each time point. Statistically significant differences are indicated $\left({ }^{*} P<0.01,{ }^{*} P<0.002\right)$. (d) Western blot analysis of AT1 receptors in aortic extracts. AT1 receptor expression in aortae from wild-type rats and $r g s 2^{+/+}(\mathrm{WT}), \mathrm{rgs} 2^{+/-}$, and $r g s 2^{-/-}$mice is indicated (upper panel). Antibody specificity was verified by probing of an identical blot with primary antibody that first had been incubated with blocking peptide (lower panel). 
a

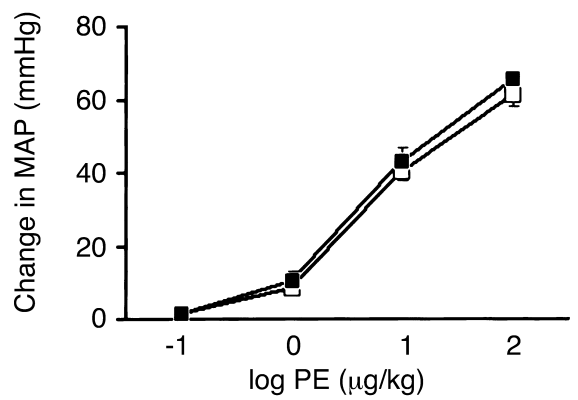

b

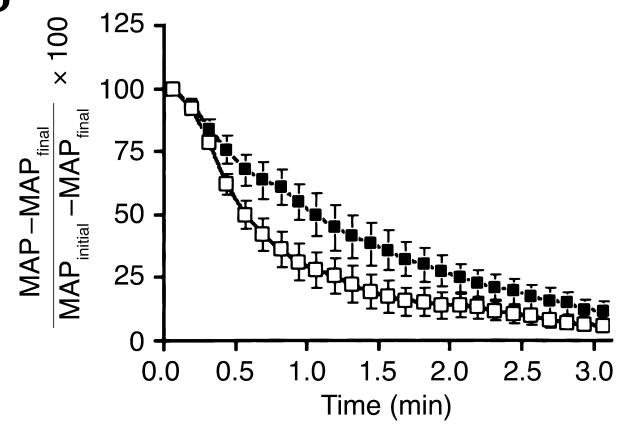

responses in vivo indicate that abnormally prolonged vasoconstrictor signaling is likely to be a significant cause of hypertension in RGS2-deficient mice. The mechanistic function of RGS2 in the vasculature therefore is analogous to that of RGS9-1 in the retina (termination of phototransduction) (34). Because loss of RGS2 prolongs responses triggered by unrelated vasoconstrictor receptors (AT1 and $\alpha_{1}$-adrenergic receptors in vivo and $\mathrm{P} 2 \mathrm{Y}$ receptors in vitro), RGS2 is likely to promote the termination of signaling by many physiologically important Gq-coupled vasoconstrictor receptors.

\section{Discussion}

In this study we have shown that signaling by $G$ protein-coupled vasoconstrictor receptors must be regulated with exquisitely precise kinetics to achieve normal

\section{Figure 5}

Changes in intracellular calcium levels in response to vasoconstrictors in primary aortic vascular smooth muscle cells derived from wild-type and $r g s 2^{-/-}$mice. (a) Dose-response relationships for P2Y receptormediated increases in intracellular calcium levels. Cells were treated with the indicated doses of ATP, an agonist for P2Y receptors. Changes in intracellular calcium levels were measured as changes in fluorescence ratio $[F R \pm S E M=($ emission at $510 \mathrm{~nm}$ upon excitation at 340 $\mathrm{nm}) /($ emission at $510 \mathrm{~nm}$ upon excitation at $380 \mathrm{~nm})]$ in fura-2loaded cells isolated from wild-type $(n=15-22$ individual cells for each agonist dose; open squares) and rgs $2^{-1-}$ mice $(n=15-22$ individual cells for each agonist dose; filled squares). (b) Rates of intracellular calcium level decay $\left(100 \% \times\left[F R-F R_{\text {final }}\right] /\left[F_{\text {initial }}-F_{\text {final }}\right] \pm S E M\right)$ after a maximal response had been elicited by treating cells from wild-type ( $n=39$ individual cells; open squares) and $r g s 2^{-/-}$mice $(n=46$ individual cells; filled squares) with $100 \mu \mathrm{M}$ ATP. Pairwise comparisons of the FR values of wild-type and rgs $2^{-/-}$cells were made at similar agonist concentrations in a or similar times after maximal FR response in b. Statistically significant differences are indicated $\left({ }^{*} P<0.001\right)$.

\section{Figure 4}

Vasoconstrictor dose-response relationships and kinetics of blood pressure decline in wild-type and rgs $2^{-/}$mice. (a) Dose-response relationships for blood pressure increase (change in MAP) upon challenge with increasing doses of the $\alpha$-adrenergic vasoconstrictor phenylephrine in wild-type ( $n=8$; open squares) and $r g s 2^{-/-}$mice $(n=7$; filled squares) that had been pretreated with candesartan to decrease initial blood pressures to similar low basal levels. (b) Rates of blood pressure decrease $\left[\left(M A P-M A P_{\text {final }}\right) /\left(M A P_{\text {initial }}-M A P_{\text {final }}\right)\right] \pm S E M$ following challenge with a pressor dose of phenylephrine in wild-type ( $n=6$; open squares) and $r g s 2^{-/-}$mice $(n=6$; filled squares) pretreated with candesartan to decrease blood pressure to low basal levels.

blood pressure homeostasis. We have reached this conclusion by analyzing mice lacking RGS2, an intracellular GTPase-activating protein that stimulates the rate of $G$ protein deactivation. Disruption of the rgs 2 gene in mice dramatically increases blood pressure and markedly prolongs vasoconstrictor responses of the peripheral resistance vasculature in vivo and of aortic vascular smooth muscle cells in vitro. Because RGS2 is a potent inhibitor of $\mathrm{Gq}$, these physiological and biochemical effects are likely to result significantly from prolonged activation of $\mathrm{Gq} \alpha$ and its effector phospholipase C- $\beta$ in vascular smooth muscle cells. Sustained activation of phospholipase C- $\beta$ would be expected to result in prolonged or elevated production of diacylglycerol and inositol-1,4,5trisphosphate, enhancing the release of intracellular calcium and consequent activation of myosin light chain kinase to trigger sustained smooth muscle cell contraction. Therefore, by prolonging the rate at which vasoconstrictor signaling terminates, loss of RGS2 is proposed to increase the duration of contraction by the resistance vasculature, significantly increasing blood pressure. This mechanism of hypertension is supported

a

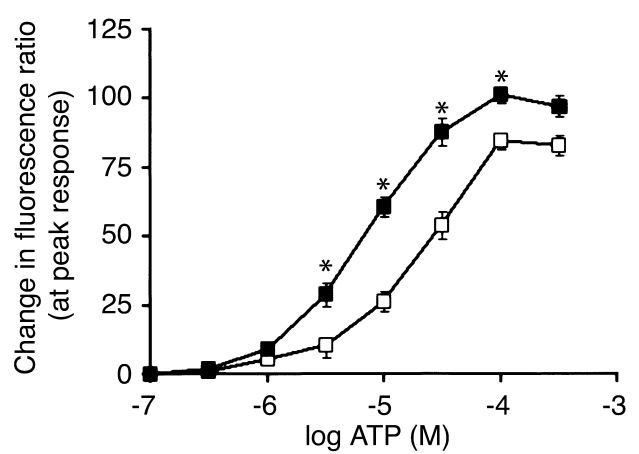

b

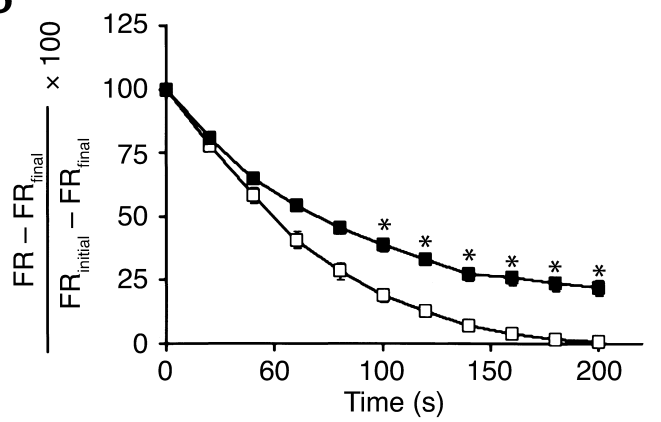


by the observations that the hypertensive phenotype of rgs $2^{-/-}$mutants is reversed within minutes following either blockade of angiotensin II production with an ACE inhibitor or antagonism of AT1 receptors, and that the rate of blood pressure decline in $\mathrm{rgs}^{-/-}$mutants infused with an AT1 antagonist is slower than that in identically treated wild-type mice.

Is kinetic dysregulation of GPCR signaling in the vasculature of $r g s 2^{-/-}$mice likely to be sufficient to cause hypertension? Although further studies will be necessary to answer this question, it is interesting that vascular overexpression of the GPCR kinase GRK2 in transgenic mice is sufficient to cause hypertension and impaired vascular $\beta$-adrenergic receptor signaling (35). Given such a precedent and the results of our study, we suggest that kinetic dysregulation of vasoconstrictor signaling in the vasculature of $r g s 2^{-/-}$mice contributes substantially to hypertension.

An unexpected finding of this study is that hypertension in $r g s 2^{--}$mice is essentially completely dependent on angiotensin II signaling. This is in contrast to previous biochemical studies, and evidence presented here, that show that RGS2 is capable of negatively regulating signaling by several types of Gq-coupled vasoconstrictor receptors $(36,37)$. The angiotensin II dependence of the hypertensive phenotype of $r g s 2^{-/-}$mice may be due significantly to the fact that maintenance of blood pressure in anesthetized wild-type animals is more strongly dependent on the renin-angiotensin system than on the sympathetic nervous system (38). Pharmacological studies combined with telemetric analysis of blood pressure in unanesthetized rgs $^{-2^{--}}$mice will be necessary to establish the relative contributions of the renin-angiotensin and sympathetic nervous systems as drivers of the hypertensive phenotype.

Another striking observation is that heterozygous and homozygous rgs2-null mice exhibit a similar level of profound hypertension. Although subtle phenotypic differences may occur, such as during aging, this observation indicates that RGS2 is especially crucial for normal blood pressure homeostasis, and that mice cannot compensate for the loss of even one copy of the rgs 2 gene. Effective compensation may fail to occur because the rgs 2 gene is expressed widely in the vasculature, brain, and kidney in a pattern that is not replicated by other $r g s$ genes $(16,18-20)$, or because other RGS proteins are less selective or potent than RGS2 as regulators of $\mathrm{Gq}$, as is suggested by biochemical studies $(36,37)$. Alternatively, RGS2 deficiency could cause developmental defects in regulatory circuitry, such as in the nervous system, that otherwise would compensate for imbalances in blood pressure regulation by controlling release and/or signaling by vasoconstrictors such as norepinephrine or angiotensin II. However, decreases in apical and basilar spines in the CA1 region of the hippocampus of $r g s 2^{-/-}$mice (19) are unlikely to be responsible, because they are not observed in $r g s 2$ heterozygous null mutants and yet these mice are hypertensive.
Lack of significant difference in the hypertensive phenotypes of $r g s 2$ heterozygous versus homozygous nulls could result from stimulation of endothelial production of $\mathrm{NO}$ or other vasodilatory factors by angiotensin II (39-42). According to this model, an initial response to angiotensin II would be more robust in rgs 2 homozygous nulls than in heterozygous nulls, but blood pressure would not be higher in rgs 2 homozygous nulls because they would produce more NO or other vasodilatory factors as a counterbalancing mechanism.

In conclusion, results of the present study and previous investigations demonstrate that hypertension in rodents can be caused by abnormalities in several components of $G$ protein signaling pathways, including receptors $(3,4)$, agonists $(5,6)$, and RGS2. This underscores the importance of GPCRs and the signals they transduce for normal blood pressure homeostasis and suggests that dysfunctional GPCR signaling may contribute significantly to the initiation, establishment, and/or maintenance of essential hypertension in humans. Indeed, polymorphisms in the genes encoding human $\beta_{1}$ - and $\beta_{2}$-adrenergic receptors have been implicated as markers associated with increased risk of hypertension (7-10). Because RGS2 and other RGS proteins that are relatively widely expressed have the potential to coordinately regulate signaling by several GPCRs involved in blood pressure homeostasis, genetic defects affecting their function or expression may constitute significant risk factors for the development of hypertension. Identification of such abnormalities that result in kinetic dysregulation of GPCR signaling therefore may lead to new means of diagnosing or treating hypertension.

\section{Acknowledgments}

We are grateful to Greg Bach for his assistance with the calcium-imaging studies. This work was supported by grants from the NIH, the National Science Foundation, and the Pharmacia/Washington University Biomedical Research Agreement. S.P. Heximer is a postdoctoral fellow of the American Heart Association.

1. Lifton, R.P., Gharavi, A.G., and Geller, D.S. 2001. Molecular mechanisms of human hypertension. Cell. 104:545-556.

2. Takahashi, N., and Smithies, O. 1999. Gene targeting approaches to analyzing hypertension. J. Am. Soc. Nephrol. 10:1598-1605.

3. Asico, L.D., et al. 1998. Disruption of the dopamine D3 receptor gene produces renin-dependent hypertension. J. Clin. Invest. 102:493-498.

4. Berthiaume, N., Yanagisawa, M., Labonte, J., and D'Orleans-Juste, P. 2000. Heterozygous knock-out of ET(B) receptors induces BQ-123-sensitive hypertension in the mouse. Hypertension. 36:1002-1007.

5. Kim, H.S., et al. 1995. Genetic control of blood pressure and the angiotensinogen locus. Proc. Natl. Acad. Sci. USA. 92:2735-2739.

6. Niranjan, V., Telemaque, S., deWit, D., Gerard, R.D., and Yanagisawa, M. 1996. Systemic hypertension induced by hepatic overexpression of human preproendothelin-1 in rats. J. Clin. Invest. 98:2364-2372.

7. Bengtsson, K., et al. 2001. Polymorphism in the beta(1)-adrenergic receptor gene and hypertension. Circulation. 104:187-190.

8. Bray, M.S., et al. 2000. Positional genomic analysis identifies the beta(2)adrenergic receptor gene as a susceptibility locus for human hypertension. Circulation. 101:2877-2882.

9. Cockcroft, J.R., et al. 2000. Beta(2)-adrenoceptor polymorphism determines vascular reactivity in humans. Hypertension. 36:371-375.

10. Hoit, B.D., Suresh, D.P., Craft, L., Walsh, R.A., and Liggett, S.B. 2000. beta2-adrenergic receptor polymorphisms at amino acid 16 differentially 
influence agonist-stimulated blood pressure and peripheral blood flow in normal individuals. Am. Heart J. 139:537-542.

11. Liolitsa, D., Powell, J.F., Prince, M., and Lovestone, S. 2001. Association study of the 5-HT(2A) receptor gene polymorphism, T102C and essential hypertension. J. Hum. Hypertens. 15:335-339.

12. Sierra, C., et al. 2002. Renin-angiotensin system genetic polymorphisms and cerebral white matter lesions in essential hypertension. Hypertension. 39:343-347.

13. Wang, J.G., and Staessen, J.A. 2000. Genetic polymorphisms in the reninangiotensin system: relevance for susceptibility to cardiovascular disease. Eur. J. Pharmacol. 410:289-302.

14. Bourne, H.R. 1997. How receptors talk to trimeric G proteins. Curr. Opin. Cell Biol. 9:134-142.

15. Ross, E.M., and Wilkie, T.M. 2000. GTPase-activating proteins for heterotrimeric $G$ proteins: regulators of $G$ protein signaling (RGS) and RGS-like proteins. Annu. Rev. Biochem. 69:795-827.

16. Ingi, T., et al. 1998. Dynamic regulation of RGS2 suggests a novel mechanism in G-protein signaling and neuronal plasticity. J. Neurosci. 18:7178-7188.

17. Sinnarajah, S., et al. 2001. RGS2 regulates signal transduction in olfactory neurons by attenuating activation of adenylyl cyclase III. Nature. 409:1051-1055.

18. Chen, C., Zheng, B., Han, J., and Lin, S.C. 1997. Characterization of a novel mammalian RGS protein that binds to Galpha proteins and inhibits pheromone signaling in yeast. J. Biol. Chem. 272:8679-8685.

19. Oliveira-Dos-Santos, A.J., et al. 2000. Regulation of T cell activation, anxiety, and male aggression by RGS2. Proc. Natl. Acad. Sci. USA. 97:12272-12277.

20. Grant, S.L., et al. 2000. Specific regulation of RGS2 messenger RNA by angiotensin II in cultured vascular smooth muscle cells. Mol. Pharmacol. 57:460-467.

21. Wu, H.K., et al. 1995. Differential expression of a basic helix-loop-helix phosphoprotein gene, G0S8, in acute leukemia and localization to human chromosome 1q31. Leukemia. 9:1291-1298.

22. Mansfield, T.A., et al. 1997. Multilocus linkage of familial hyperkalaemia and hypertension, pseudohypoaldosteronism type II, to chromosomes 1q31-42 and 17p11-q21. Nat. Genet. 16:202-205.

23. Lorenz, J.N., and Kranias, E.G. 1997. Regulatory effects of phospholamban on cardiac function in intact mice. Am. J. Physiol. 273:H2826-H2831.

24. Mozaffari, M.S., Roysommuti, S., and Wyss, J.M. 1996. Contribution of the sympathetic nervous system to hypertensive response to insulin excess in spontaneously hypertensive rats. J. Cardiovasc. Pharmacol. 27:539-544.

25. Rogers, J.H., et al. 1999. RGS4 causes increased mortality and reduced cardiac hypertrophy in response to pressure overload. J. Clin. Invest. 104:567-576.

26. Hayashi, K., et al. 1999. Changes in the balance of phosphoinositide 3-kinase/protein kinase $\mathrm{B}(\mathrm{Akt})$ and the mitogen-activated protein kinases
(ERK/p38MAPK) determine a phenotype of visceral and vascular smooth muscle cells. J. Cell Biol. 145:727-740.

27. Johns, C., Gavras, I., Handy, D.E., Salomao, A., and Gavras, H. 1996. Models of experimental hypertension in mice. Hypertension. 28:1064-1069.

28. Intengan, H.D., and Schiffrin, E.L. 2001. Vascular remodeling in hypertension: roles of apoptosis, inflammation, and fibrosis. Hypertension. 38:581-587.

29. Bivalacqua, T.J., Dalal, A., Champion, H.C., and Kadowitz, P.J. 1999. Role of AT(1) receptors and autonomic nervous system in mediating acute pressor responses to ANG II in anesthetized mice. Am. J. Physiol. 277:E838-E847.

30. Gratton, J.P., et al. 1995. Pharmacological properties of endothelins and big endothelins in ketamine/xylazine or urethane anesthetized rats. Am. J. Hypertens. 8:1121-1127.

31. Ma, X., Abboud, F.M., and Chapleau, M.W. 2001. A novel effect of angiotensin on renal sympathetic nerve activity in mice. J. Hypertens. 19:609-618.

32. Duka, I., Gavras, I., Johns, C., Handy, D.E., and Gavras, H. 2000. Role of the postsynaptic alpha(2)-adrenergic receptor subtypes in catecholamine-induced vasoconstriction. Gen. Pharmacol. 34:101-106.

33. Erlinge, D. 1998. Extracellular ATP: a growth factor for vascular smooth muscle cells. Gen. Pharmacol. 31:1-8.

34. Chen, C.K., et al. 2000. Slowed recovery of rod photoresponse in mice lacking the GTPase accelerating protein RGS9-1. Nature. 403:557-560.

35. Eckhart, A.D., Ozaki, T., Tevaearai, H., Rockman, H.A., and Koch, W.J. 2002. Vascular-targeted overexpression of $G$ protein-coupled receptor kinase- 2 in transgenic mice attenuates beta-adrenergic receptor signaling and increases resting blood pressure. Mol. Pharmacol. 61:749-758.

36. Heximer, S.P., Watson, N., Linder, M.E., Blumer, K.J., and Hepler, J.R. 1997. RGS2/G0S8 is a selective inhibitor of Gqalpha function. Proc. Natl. Acad. Sci. USA 94:14389-14393.

37. Heximer, S.P., et al. 1999. G protein selectivity is a determinant of RGS2 function. J. Biol. Chem. 274:34253-34259.

38. Ullman, J. 1999. Vasopressin and angiotensin II in blood pressure control during isoflurane anesthesia in rats. Acta Anaesthesiol. Scand. 43:860-865.

39. Saito, S., Hirata, Y., Emori, T., Imai, T., and Marumo, F. 1996. Angiotensin II activates endothelial constitutive nitric oxide synthase via AT1 receptors. Hypertens. Res. 19:201-206.

40. Hennington, B.S., Zhang, H., Miller, M.T., Granger, J.P., and Reckelhoff, J.F. 1998. Angiotensin II stimulates synthesis of endothelial nitric oxide synthase. Hypertension. 31:283-288.

41. Pueyo, M.E., Arnal, J.F., Rami, J., and Michel, J.B. 1998. Angiotensin II stimulates the production of NO and peroxynitrite in endothelial cells. Am. J. Physiol. 274:C214-C220.

42. Tsutsumi, Y., et al. 1999. Angiotensin II type 2 receptor overexpression activates the vascular kinin system and causes vasodilation. J. Clin. Invest. 104:925-935. 